\title{
Gap Function Approach to the Generalized Nash Equilibrium Problem
}

\author{
Katsunori Kubota and Masao Fukushima \\ Department of Applied Mathematics and Physics \\ Graduate School of Informatics \\ Kyoto University \\ Kyoto 606-8501, Japan
}

April 3, 2009, revised July 27, 2009

\begin{abstract}
We consider an optimization reformulation approach for the generalized Nash equilibrium problem (GNEP) that uses the regularized gap function of a quasi-variational inequality (QVI). The regularized gap function for QVI is in general not differentiable, but only directionally differentiable. Moreover, a simple condition has yet to be established, under which any stationary point of the regularized gap function solves the QVI. We tackle these issues for the GNEP in which the shared constraints are given by linear equalities, while the individual constraints are given by convex inequalities. First, we formulate the minimization problem involving the regularized gap function, and show the equivalence to GNEP. Next, we establish the differentiability of the regularized gap function and show that any stationary point of the minimization problem solves the original GNEP under some suitable assumptions. Then, by using a barrier technique, we propose an algorithm that sequentially solves minimization problems obtained from GNEPs with the shared equality constraints only. Further, we discuss the case of shared inequality constraints and present an algorithm that utilizes the transformation of the inequality constraints to equality constraints by means of slack variables. We present some results of numerical experiments to illustrate the proposed approach.
\end{abstract}

Keywords. Generalized Nash equilibrium problem, quasi-variational inequality, regularized gap function.

AMS Classifications. 65K05, 90C30, 91A10.

\section{Introduction}

A multi-player non-cooperative game is called the Nash equilibrium problem (NEP), if the goal is to find a solution in which no player has any incentive to change his/her own strategy unilaterally. The generalized Nash equilibrium problem (GNEP) is a generalization of the NEP, in which each player's strategy set depends on the other players' strategies as well [1]. A solution of the GNEP is called a generalized Nash equilibrium (GNE). The GNEP has many applications such as electric power market models $[2,3]$ and river basin pollution games $[4,5,6]$.

Recently, an increasing effort has been made to develop algorithms for computing GNEs [1]. Some of them are based on the well-known fact that a NEP can be reformulated as a variational inequality (VI) if each player's problem is a convex programming problem $[7,8,9]$. Pang and Fukushima [3] proposed an approach for GNEP that solves a sequence of VIs corresponding to 
NEPs, which are obtained by approximating the original GNEP by means of a penalty technique. Along a similar line, Facchinei and Pang [10] proposed to use an exact penalty function. More recently, Fukushima [11] proposed a controlled penalty method to find a particular GNE called a restricted GNE that contains a normalized equilibrium of Rosen [12] as a special case.

Besides penalty methods, several algorithms have been proposed for GNEPs. Facchinei, Fischer and Piccialli [13] studied Newton-type methods for finding a normalized equilibrium by way of a VI reformulation of the GNEP with shared constraints, while von Heusinger, Kanzow and Fukushima [14] proposed a generalized Newton method applied to a fixed point problem derived from the original GNEP. Nabetani, Tseng and Fukushima [15] proposed parametrized VI approaches to GNEP, with particular emphasis on finding GNEs as many as possible. Krawczyk and Uryasev [6] and von Heusinger and Kanzow [16], among others, proposed Nikaido-Isoda function-type approaches to compute GNEs.

Yet another approach is based on the link between a GNEP and a quasi-variational inequality (QVI). It is known that a GNEP can be reformulated as a QVI under some assumptions $[17,3]$. The relationship between the GNEP and QVI in Hilbert space was studied by Bensoussan [18]. Harker [17] obtained some results for problems in a finite-dimensional Euclidean space. However, compared with the VI, the study of the QVI is still in its infancy, and only a few algorithms have been proposed to solve QVIs numerically. Fukushima [19] defined the regularized gap function for a QVI, which is an extension of the one for a VI [20], and showed that the QVI can be solved by minimizing the regularized gap function. However, there still remain some difficulties with this approach. Unlike the case of VI, the regularized gap function for QVI is in general not differentiable, but only directionally differentiable [19]. Moreover, for VI, under some monotonicity assumption, it is proved that any stationary point of the regularized gap function solves the VI [19]. However, such a simple condition for the QVI has yet to be established.

In this paper, we focus on the GNEP in which the shared constraints are given by linear equalities, while the individual constraints are given by convex inequalities. First, we formulate the minimization problem with the regularized gap function, and show the equivalence between this minimization problem and the GNEP. Next, we establish the differentiability of the regularized gap function and show that any stationary point of the minimization problem solves the original GNEP under suitable assumptions. This is the main theoretical contribution of the paper. Then, by using a barrier technique, we propose an algorithm that sequentially solves minimization problems obtained from GNEPs with the shared equality constraints only. Further, we discuss the case of shared inequality constraints and present an algorithm that utilizes the transformation of inequality constraints to equality constraints by means of slack variables. Finally, we present some results of numerical experiments to illustrate the proposed approach.

We use the following notation throughout the paper. For vectors $x, y \in \mathbb{R}^{n}$, the inner product is denoted by $\langle x, y\rangle:=x^{\top} y$, where ${ }^{\top}$ denotes transposition. For a vector $x \in \mathbb{R}^{n}$, the Euclidean norm is denoted by $\|x\|:=\sqrt{\langle x, x\rangle}$. For a transposed vector comprised of several subvectors, we use a simplified notation $\left(x^{1}, x^{2}, \ldots, x^{N}\right)^{\top}$ instead of $\left(\left(x^{1}\right)^{\top},\left(x^{2}\right)^{\top}, \ldots,\left(x^{N}\right)^{\top}\right)^{\top}$. 


\section{Generalized Nash Equilibrium Problem}

Consider an $N$-person non-cooperative game in which each player's strategy set depends on the other players' strategies. Specifically, let each player $\nu$ solve the following optimization problem for $x^{\nu}$ with $x^{-\nu}$ treated as exogenous:

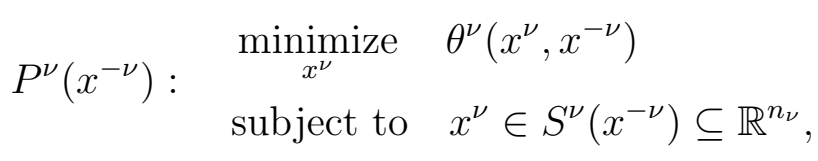

where

$$
x:=\left(x^{\nu}\right)_{\nu=1}^{N} \in \mathbb{R}^{n}, \quad x^{-\nu}:=\left(x^{\nu^{\prime}}\right)_{\nu^{\prime}=1, \nu^{\prime} \neq \nu}^{N} \in \mathbb{R}^{n_{-\nu}}, \quad n:=\sum_{\nu=1}^{N} n_{\nu}, \quad n_{-\nu}:=n-n_{\nu} .
$$

Here, $x^{\nu} \in \mathbb{R}^{n_{\nu}}$ denotes the strategy of player $\nu$, and $x^{-\nu} \in \mathbb{R}^{n_{-\nu}}$ denotes the vector formed by the strategies of all players except player $\nu$. The objective function $\theta^{\nu}: \mathbb{R}^{n_{\nu}} \times \mathbb{R}^{n_{-\nu}} \rightarrow \mathbb{R}$ of player $\nu$ is assumed to be a differentiable convex function for any fixed $x^{-\nu}$. Player $\nu$ 's strategy set $S^{\nu}\left(x^{-\nu}\right) \subseteq \mathbb{R}^{n_{\nu}}$ is a convex set, and depends on the other player's strategies. Thus, each player's problem is a convex programming problem.

A GNE is then defined to be a tuple $x^{*}:=\left(x^{*, \nu}\right)_{\nu=1}^{N}$ such that $x^{*, \nu}$ is an optimal solution of the following optimization problem for each $\nu=1, \ldots, N$ :

$$
\begin{array}{lll}
P^{\nu}\left(x^{*,-\nu}\right): & \underset{x^{\nu}}{\operatorname{minimize}} & \theta^{\nu}\left(x^{\nu}, x^{*,-\nu}\right) \\
& \text { subject to } & x^{\nu} \in S^{\nu}\left(x^{*,-\nu}\right) .
\end{array}
$$

This means that, when each player $\nu$ chooses the strategy $x^{*, \nu}$, no player has any incentive to change his/her strategy unilaterally.

In particular, if each player's strategy set does not depend on the other players' strategies, then a GNE reduces to the classical Nash equilibrium.

\section{Reformulation of GNEP as QVI}

Define the vector-valued function $F: \mathbb{R}^{n} \rightarrow \mathbb{R}^{n}$ and the point-to-set mapping $S: \mathbb{R}^{n} \rightrightarrows \mathbb{R}^{n}$ by

$$
\begin{aligned}
F(x) & :=\left(F^{\nu}(x)\right)_{\nu=1}^{N}:=\left(\nabla_{x^{\nu}} \theta^{\nu}\left(x^{\nu}, x^{-\nu}\right)\right)_{\nu=1}^{N} \in \mathbb{R}^{n}, \\
S(x) & :=\prod_{\nu=1}^{N} S^{\nu}\left(x^{-\nu}\right) \subseteq \mathbb{R}^{n} .
\end{aligned}
$$

By assumption, problem (1) is a convex programming problem for each $\nu$. Therefore, $x^{*, \nu}$ is an optimal solution of (1) if and only if $x^{*, \nu}$ is a stationary point of the function $\theta^{\nu}\left(\cdot, x^{*,-\nu}\right)$ on the set $S\left(x^{*,-\nu}\right)$, that is, $x^{*, \nu}$ satisfies

$$
x^{*, \nu} \in S^{\nu}\left(x^{*,-\nu}\right)
$$


and

$$
\left\langle\nabla_{x^{\nu}} \theta^{\nu}\left(x^{*, \nu}, x^{*,-\nu}\right), x^{\nu}-x^{*, \nu}\right\rangle \geq 0, \quad \forall x^{\nu} \in S\left(x^{*,-\nu}\right) .
$$

Thus, the GNEP defined in Section 2 is equivalent to finding a vector $x^{*} \in \mathbb{R}^{n}$ such that $x^{*} \in S\left(x^{*}\right)$ and

$$
\left\langle F\left(x^{*}\right), y-x^{*}\right\rangle \geq 0, \quad \forall y \in S\left(x^{*}\right)
$$

This type of problem is called a quasi-variational inequality (QVI). In particular, if $S(x)=\hat{S}$ for all $x$, where $\hat{S}$ is a nonempty closed convex set, then QVI (3) reduces to a variational inequality (VI).

\section{A Merit Function for QVI}

Generally, a merit function of an equilibrium problem refers to a nonnegative-valued function $f$ such that $x$ is a solution of the problem if and only if $f(x)=0$ and $x$ satisfies the constraints of the problem. The equilibrium problem can be reformulated as an equivalent optimization problem by means of a merit function. For VIs, there have been several proposals of merit functions, such as the gap function [21] and the regularized gap function [20], and the properties of those functions have been studied extensively.

For QVI (3), an extension of the regularized gap function is proposed by Fukushima [19]. This function, also called the regularized gap function for the QVI, is defined by

$$
f(x):=-\inf _{y}\left\{\langle F(x), y-x\rangle+\frac{1}{2}\langle y-x, H(y-x)\rangle \mid y \in S(x)\right\},
$$

where $H$ is a symmetric positive definite matrix. Let the set $X \subseteq \mathbb{R}^{n}$ be defined by

$$
X:=\left\{x \in \mathbb{R}^{n} \mid x \in S(x)\right\},
$$

which is called the feasible set of QVI (3). Similarly to VI, for any $x \in X$, the minimization problem on the right-hand side of (4) is a convex programming problem, and it has a unique optimal solution for any $x$. We denote this optimal solution by $y(x)$. Then the regularized gap function $f$ is written as

$$
f(x)=-\langle F(x), y(x)-x\rangle-\frac{1}{2}\langle y(x)-x, H(y(x)-x)\rangle .
$$

The following result holds [19].

Theorem 4.1. For each $x \in X$, we have $f(x) \geq 0$. Moreover, $x$ solves $Q V I$ (3) if and only if $f(x)=0$ and $x \in X$.

This theorem indicates that QVI (3) can be reformulated as the following optimization problem:

$$
Q: \begin{array}{ll}
\text { minimize } & f(x) \\
& \text { subject to } \quad x \in X
\end{array}
$$


That is, the function $f$ is a merit function of QVI (3). Unfortunately, unlike the case of VI, the regularized gap function $f$ for QVI is in general not differentiable, but only directionally differentiable when the function $F$ is differentiable. Moreover, even if $\nabla F(x)$ is positive definite at any stationary point $x$ of problem $Q$, it does not imply that $x$ solves the original QVI. In the next section, we will explore the possibility of avoiding these difficulties with the gap function approach for a class of GNEPs.

\section{GNEP with Shared Equality Constraints and Barrier Method}

Consider the GNEP with player $\nu$ 's problem:

$$
\begin{array}{cl}
\underset{x^{\nu}}{\operatorname{minimize}} & \theta^{\nu}\left(x^{\nu}, x^{-\nu}\right) \\
\text { subject to } & \left\langle a_{i}^{\nu}, x^{\nu}\right\rangle=b_{i}-\sum_{\nu^{\prime} \neq \nu}\left\langle a_{i}^{\nu^{\prime}}, x^{\nu^{\prime}}\right\rangle, \quad i=1, \ldots, m, \\
& h_{j}^{\nu}\left(x^{\nu}\right) \leq 0, \quad j=1, \ldots, l_{\nu} .
\end{array}
$$

Notice that the shared constraints are given by equalities, while the individual constraints are given by inequalities. We denote this GNEP as $P$.

In the remainder of this paper, we make the following assumption:

Assumption 5.1. $\theta^{\nu}\left(\cdot, x^{-\nu}\right): \mathbb{R}^{n_{\nu}} \rightarrow \mathbb{R}$ is a twice continuously differentiable convex function for any fixed $x^{-\nu} \in \mathbb{R}^{n_{-\nu}}$, and $h_{j}^{\nu}: \mathbb{R}^{n_{\nu}} \rightarrow \mathbb{R}, j=1, \ldots, l_{\nu}$ are twice continuously differentiable convex functions.

We apply a barrier technique to the individual inequality constraints, thereby reformulating the GNEP into another GNEP with the shared equality constraints only. We then develop an optimization approach using the regularized gap function for the QVI derived from the latter GNEP. Note that the proposed barrier method incorporates each player's individual constraints in the objective function by using the barrier function. This is different from the common approach where the penalty technique is applied to the shared constraints $[10,11,3]$.

By adding the barrier term associated with the individual constraints to the objective function, problem (5) is approximated by the following problem:

$$
\begin{array}{ll}
\underset{x^{\nu}}{\operatorname{minimize}} & \theta^{\nu}\left(x^{\nu}, x^{-\nu}\right)-\rho \sum_{j=1}^{l_{\nu}} \log \left(-h_{j}^{\nu}\left(x^{\nu}\right)\right) \\
\text { subject to } & \left\langle a_{i}^{\nu}, x^{\nu}\right\rangle=b_{i}-\sum_{\nu^{\prime} \neq \nu}\left\langle a_{i}^{\nu^{\prime}}, x^{\nu^{\prime}}\right\rangle, \quad i=1, \ldots, m,
\end{array}
$$

where $\rho>0$ is a barrier parameter. Let $P_{\rho}$ denote the GNEP with each player's problem given by (6). Since problem (6) is a convex programming problem, GNEP $P_{\rho}$ can be reformulated as the following QVI: Find a vector $x \in S_{0}(x) \cap \Sigma_{0}$ such that

$$
\langle F(x)-\rho E(x), y-x\rangle \geq 0, \quad \forall y \in S_{0}(x)
$$


where the function $F$ is defined by (2), and $\Sigma_{0} \subseteq \mathbb{R}^{n}, E: \Sigma_{0} \rightarrow \mathbb{R}^{n}$ and $S_{0}: \mathbb{R}^{n} \rightrightarrows \mathbb{R}^{n}$ are defined by

$$
\begin{aligned}
\Sigma_{0} & :=\prod_{\nu=1}^{N}\left\{x^{\nu} \mid h_{j}^{\nu}\left(x^{\nu}\right)<0, j=1, \ldots, l_{\nu}\right\}, \\
E(x) & :=\left(\sum_{j=1}^{l_{\nu}} \frac{\nabla h_{j}^{\nu}\left(x^{\nu}\right)}{h_{j}^{\nu}\left(x^{\nu}\right)}\right)_{\nu=1}^{N}, \\
S_{0}(x) & :=\prod_{\nu=1}^{N}\left\{y^{\nu} \mid\left\langle a_{i}^{\nu}, y^{\nu}\right\rangle=b_{i}-\sum_{\substack{\nu^{\prime}=1 \\
\nu^{\prime} \neq \nu}}^{N}\left\langle a_{i}^{\nu^{\prime}}, x^{\nu^{\prime}}\right\rangle, i=1, \ldots, m\right\},
\end{aligned}
$$

respectively. Then, the regularized gap function for this problem is defined by

$$
f_{\rho}(x):=-\inf \left\{\langle F(x)-\rho E(x), y-x\rangle+\frac{1}{2}\langle y-x, H(y-x)\rangle \mid y \in S_{0}(x)\right\} .
$$

Note that the function $f_{\rho}$ is defined only on the open set $\Sigma_{0}$. By letting $f_{\rho}(x)=+\infty \forall x \notin \Sigma_{0}$, GNEP $P_{\rho}$ is reformulated as the minimization problem

$$
\begin{array}{lll}
Q_{\rho}: & \text { minimize } & f_{\rho}(x) \\
& \text { subject to } & x \in X_{0}
\end{array}
$$

where the set $X_{0} \subseteq \mathbb{R}^{n}$ is defined by

$$
X_{0}:=\left\{x \mid b_{i}-\sum_{\nu=1}^{N}\left\langle a_{i}^{\nu}, x^{\nu}\right\rangle=0, i=1, \ldots, m\right\} .
$$

This fact is formally stated as follows.

Theorem 5.1. For each $x \in X_{0}$, we have $f_{\rho}(x) \geq 0$. Moreover, $x$ solves $Q V I$ (7) if and only if $f_{\rho}(x)=0$ and $x \in X_{0}$.

Now we consider the differentiability of the function $f_{\rho}$. Let $y(x)$ denote the unique solution of the optimization problem on the right-hand side of $(9)$.

Lemma 5.1. If $x \in X_{0}$, then $y(x) \in X_{0}$.

Proof. Since $y(x) \in S_{0}(x)$, for each $\nu=1, \ldots, N, y^{\nu}(x)$ satisfies

$$
\left\langle a_{i}^{\nu}, y^{\nu}(x)\right\rangle=b_{i}-\sum_{\nu^{\prime} \neq \nu}\left\langle a_{i}^{\nu^{\prime}}, x^{\nu^{\prime}}\right\rangle, \quad i=1, \ldots, m .
$$

Since $x \in X_{0}$ by assumption, we have

$$
b_{i}-\sum_{\nu=1}^{N}\left\langle a_{i}^{\nu}, x^{\nu}\right\rangle=0, \quad i=1, \ldots, m .
$$


Hence, by (10) and (11), for each $\nu=1, \ldots, N$, we obtain

$$
\left\langle a_{i}^{\nu}, x^{\nu}\right\rangle=\left\langle a_{i}^{\nu}, y^{\nu}(x)\right\rangle, \quad i=1, \ldots, m
$$

Therefore, by (11) and (12), we have

$$
0=b_{i}-\sum_{\nu=1}^{N}\left\langle a_{i}^{\nu}, x^{\nu}\right\rangle=b_{i}-\sum_{\nu=1}^{N}\left\langle a_{i}^{\nu}, y^{\nu}(x)\right\rangle, \quad i=1, \ldots, m,
$$

which implies $y(x) \in X_{0}$.

The following theorem shows that the function $f_{\rho}$ is directionally differentiable in general; moreover it is differentiable under suitable assumptions.

Theorem 5.2. The function $f_{\rho}$ defined by (9) is directionally differentiable at every $x \in \Sigma_{0}$ along any direction $d \in \mathbb{R}^{n}$, and the directional derivative is given by

$$
\begin{aligned}
f_{\rho}^{\prime}(x ; d)=\min _{\mu \in M(x)}\{ & \langle(F(x)-\rho E(x))-(\nabla F(x)-\rho \nabla E(x)-H)(y(x)-x), d\rangle \\
& \left.-\sum_{i=1}^{m} \sum_{\nu=1}^{N} \mu_{i}^{\nu}\left\langle\left(a_{i}^{1}, \ldots, a_{i}^{\nu-1}, 0, a_{i}^{\nu+1}, \ldots, a_{i}^{N}\right)^{\top}, d\right\rangle\right\},
\end{aligned}
$$

where $M(x) \subseteq \mathbb{R}^{N m}$ consists of all vectors $\mu:=\left(\left(\mu_{i}^{\nu}\right)_{i=1}^{m}\right)_{\nu=1}^{N} \in \mathbb{R}^{N m}$ satisfying

$$
F(x)-\rho E(x)+H(y(x)-x)+\sum_{i=1}^{m} \sum_{\nu=1}^{N} \mu_{i}^{\nu}\left(0, \ldots, 0, a_{i}^{\nu}, 0, \ldots, 0\right)^{\top}=0 .
$$

In particular, if $M(x)$ is a singleton, i.e.,

$$
M(x)=\{\mu(x)\}
$$

then $f_{\rho}$ is differentiable at $x$ and the gradient of $f_{\rho}$ at $x$ is given by

$$
\begin{aligned}
\nabla f_{\rho}(x)= & (F(x)-\rho E(x))-(\nabla F(x)-\rho \nabla E(x)-H)(y(x)-x) \\
& -\sum_{i=1}^{m} \sum_{\nu=1}^{N} \mu_{i}^{\nu}(x)\left(a_{i}^{1}, \ldots, a_{i}^{\nu-1}, 0, a_{i}^{\nu+1}, \ldots, a_{i}^{N}\right)^{\top} .
\end{aligned}
$$

Proof. The regularized gap function $f_{\rho}$ is defined by substituting $F(x)-\rho E(x)$ for $F(x)$ in the definition (4) of the regularized gap function $f_{\rho}$. Since $F(x)-\rho E(x)$ is differentiable, the assertion of this theorem immediately follows from [19, Theorem 3].

The next assumption ensures that the set $M(x)$ is a singleton for any $x$. This assumption seems to be reasonable. In fact, if it fails to hold, then a player's problem (5) will become infeasible, or very unstable even if it is feasible. 
Assumption 5.2. For each $\nu=1, \ldots, N$, the vectors $a_{i}^{\nu}, i=1, \ldots, m$ are linearly independent.

Theorem 5.3. Let Assumption 5.2 hold. Then the function $f_{\rho}$ is differentiable at any point $x \in \Sigma_{0}$, and its gradient is given by

$$
\nabla f_{\rho}(x)=-(\nabla F(x)-\rho \nabla E(x))(y(x)-x)-\sum_{i=1}^{m} \sum_{\nu=1}^{N} \mu_{i}^{\nu}(x) a_{i},
$$

where $a_{i}:=\left(a_{i}^{1}, \ldots, a_{i}^{N}\right)^{\top} \in \mathbb{R}^{n}$.

Proof. By Assumption 5.2, the vectors $a_{i}^{\nu}, i=1, \ldots, m$ are linearly independent for each $\nu=1, \ldots, N$. Therefore, the vectors $\left(0, \ldots, 0, a_{i}^{\nu}, 0, \ldots, 0\right)^{\top} \in \mathbb{R}^{n}, i=1, \ldots, m, \nu=1, \ldots, N$ are linearly independent, and $M(x)$ has only one element $\mu(x)$. Hence, by Theorem 5.2, the function $f_{\rho}$ is differentiable at any point $x \in \Sigma_{0}$.

Moreover, by Theorem 5.2, the gradient of $f_{\rho}$ at $x$ is given by

$$
\begin{aligned}
\nabla f_{\rho}(x)= & (F(x)-\rho E(x))-(\nabla F(x)-\rho \nabla E(x)-H)(y(x)-x) \\
& -\sum_{i=1}^{m} \sum_{\nu=1}^{N} \mu_{i}^{\nu}(x)\left(a_{i}^{1}, \ldots, a_{i}^{\nu-1}, 0, a_{i}^{\nu+1}, \ldots, a_{i}^{N}\right)^{\top} .
\end{aligned}
$$

The last term on the right-hand side of (14) is rewritten as

$$
\begin{aligned}
& \sum_{i=1}^{m} \sum_{\nu=1}^{N} \mu_{i}^{\nu}(x)\left(a_{i}^{1}, \ldots, a_{i}^{\nu-1}, 0, a_{i}^{\nu+1}, \ldots, a_{i}^{N}\right)^{\top} \\
= & \sum_{i=1}^{m} \sum_{\nu=1}^{N} \mu_{i}^{\nu}(x)\left(a_{i}^{1}, \ldots, a_{i}^{\nu-1}, a_{i}^{\nu}, a_{i}^{\nu+1}, \ldots, a_{i}^{N}\right)^{\top}-\sum_{i=1}^{m} \sum_{\nu=1}^{N} \mu_{i}^{\nu}(x)\left(0, \ldots, 0, a_{i}^{\nu}, 0, \ldots, 0\right)^{\top} \\
= & \sum_{i=1}^{m} \sum_{\nu=1}^{N} \mu_{i}^{\nu}(x) a_{i}+F(x)-\rho E(x)+H(y(x)-x),
\end{aligned}
$$

where the last equality follows from (13). By using (15), the formula (14) can be rewritten as

$$
\nabla f_{\rho}(x)=-(\nabla F(x)-\rho \nabla E(x))(y(x)-x)-\sum_{i=1}^{m} \sum_{\nu=1}^{N} \mu_{i}^{\nu}(x) a_{i} .
$$

The following theorem gives a condition under which any point that satisfies the first-order optimality condition for the optimization problem $Q_{\rho}$ is a solution of GNEP $P_{\rho}$.

Theorem 5.4. Suppose Assumption 5.2 holds. Let $x \in X_{0}$ be a stationary point of problem $Q_{\rho}$, and $\nabla F(x)-\rho \nabla E(x)$ be positive definite. Then $x$ is a solution of $Q V I(7)$, i.e., $x$ is a solution of GNEP $P_{\rho}$. 
Proof. First, note that $x \in \Sigma_{0}$. By Theorem 5.3, the function $f_{\rho}$ is differentiable at the point $x$ under the given assumptions. Thus, when $x$ is a stationary point of problem $Q_{\rho}$, by making use of the fact that the feasible set $X_{0}$ is an affine set, we have

$$
\left\langle\nabla f_{\rho}(x), y-x\right\rangle=0, \quad \forall y \in X_{0}
$$

Note that $\left\langle a_{i}, x\right\rangle-b_{i}=0$ holds by $x \in X_{0}$. Moreover, we have $y(x) \in X_{0}$ by Lemma 5.1, i.e.,

$$
b_{i}-\left\langle a_{i}, y(x)\right\rangle=0, \quad i=1, \ldots, m .
$$

Hence, we have

$$
\left\langle a_{i}, y(x)-x\right\rangle=0, \quad i=1, \ldots, m .
$$

Thus, it follows from Theorem 5.3 together with (16) and (17) that

$$
\begin{aligned}
0 & =\left\langle\nabla f_{\rho}(x), y(x)-x\right\rangle \\
& =-\left\langle(\nabla F(x)-\rho \nabla E(x))(y(x)-x)+\sum_{i=1}^{m} \sum_{\nu=1}^{N} \mu_{i}^{\nu}(x) a_{i}, y(x)-x\right\rangle \\
& =-\langle(\nabla F(x)-\rho \nabla E(x))(y(x)-x), y(x)-x\rangle-\sum_{i=1}^{m} \sum_{\nu=1}^{N} \mu_{i}^{\nu}(x)\left\langle a_{i}, y(x)-x\right\rangle \\
& =-\langle(\nabla F(x)-\rho \nabla E(x))(y(x)-x), y(x)-x\rangle .
\end{aligned}
$$

Moreover, since $\nabla F(x)-\rho \nabla E(x)$ is positive definite by assumption, we must have $y(x)=x$ from (18). Then, the definition (9) of $f_{\rho}$ yields $f_{\rho}(x)=0$, and $x$ is a solution of QVI (7) according to Theorem 5.1.

Corollary 5.1. Suppose Assumption 5.2 holds and $\nabla F(x)$ is positive definite at any point $x \in X_{0} \cap \Sigma_{0}$. Then, for any $\rho>0$, a stationary point $x$ of problem $Q_{\rho}$ is a solution of GNEP $P_{\rho}$.

Proof. By direct calculation, it follows from the definition (8) of $E(x)$ that

$$
\nabla E(x)=\operatorname{Diag}\left[\sum_{j=1}^{l_{\nu}}\left(\frac{\nabla^{2} h_{j}^{\nu}\left(x^{\nu}\right)}{h_{j}^{\nu}\left(x^{\nu}\right)}-\frac{\nabla h_{j}^{\nu}\left(x^{\nu}\right) \nabla h_{j}^{\nu}\left(x^{\nu}\right)^{\top}}{h_{j}^{\nu}\left(x^{\nu}\right)^{2}}\right)\right]_{\nu=1}^{N},
$$

where $\operatorname{Diag}\left[B_{\nu}\right]_{\nu=1}^{N}$ denotes the block diagonal matrix whose block diagonal elements are $B_{\nu}, \nu=$ $1, \ldots, N$. Notice that each $\nabla^{2} h_{j}^{\nu}\left(x^{\nu}\right)$ is positive semidefinite since $h_{j}^{\nu}$ is convex. This implies that $\nabla E(x)$ is negative semidefinite for any $x \in \Sigma_{0}$, since $h_{j}^{\nu}\left(x^{\nu}\right)<0$.

Hence, $\nabla F(x)-\rho \nabla E(x)$ is positive definite at any $x \in \Sigma_{0}$, whenever so is $\nabla F(x)$. Therefore, Theorem 5.4 ensures that, for any $\rho>0$, a stationary point of $Q_{\rho}$ is a solution of GNEP $P_{\rho}$. 


\section{Convergence of the Barrier Method}

In the previous section, we have shown that, for every fixed $\rho>0$, a solution of GNEP $P_{\rho}$ can be obtained by solving the minimization problem $Q_{\rho}$. Here we present an algorithm for solving GNEP $P$ by solving problems $Q_{\rho}$ sequentially by letting the parameter $\rho$ tend to zero.

Algorithm 6.1. Choose a positive sequence $\left\{\rho_{k}\right\} \subset \mathbb{R}$ tending to zero. For each $k$, find $a$ stationary point $x_{k}$ of the minimization problem

$$
\begin{array}{lll}
Q_{\rho_{k}}: & \text { minimize } & f_{\rho_{k}}(x) \\
& \text { subject to } \quad x \in X_{0}
\end{array}
$$

By imposing appropriate conditions, we can show that the sequence $\left\{x_{k}\right\}$ generated by Algorithm 6.1 converges to a solution of GNEP P.

Assumption 6.1. $\nabla F(x)$ is positive definite at any point $x \in X_{0} \cap \Sigma_{0}$.

In view of the definition (2) of $F$, this assumption amounts to requiring the strict monotonicity of the function $F$, which is also called the diagonal strict convexity ${ }^{1}$ of the "pseudo gradient' in the context of GNEP [12]. In words, this condition implies that each player's objective function $\theta^{\nu}\left(x^{\nu}, x^{-\nu}\right)$ is strongly convex in his/her own variable $x^{\nu}$ and depends relatively less on the other players' variables $x^{-\nu}$, which seems to be a reasonable requirement.

Theorem 6.1. Suppose that Assumptions 5.2 and 6.1 hold, and the set $\Sigma^{\nu}:=\left\{x^{\nu} \in \mathbb{R}^{n_{\nu}} \mid\right.$ $\left.h^{\nu}\left(x^{\nu}\right) \leq 0\right\}$ is bounded for each $\nu=1, \ldots, N$. Let $x_{\infty}$ be any accumulating point of the sequence $\left\{x_{k}\right\}$ generated by Algorithm 6.1. Suppose the following Mangasarian-Fromovotz constraint qualification (MFCQ) holds for each $\nu=1, \ldots, N$ :

$$
\left.\begin{array}{c}
\sum_{j \in \gamma_{\infty}^{\nu}} \lambda_{j}^{\nu} \nabla h_{j}^{\nu}\left(x_{\infty}^{\nu}\right)+\sum_{i=1}^{m} \mu_{i}^{\nu} a_{i}^{\nu}=0 \\
\lambda_{j}^{\nu} \geq 0, \quad j \in \gamma_{\infty}^{\nu} \\
\mu_{i}^{\nu} \in \mathbb{R}, \quad i=1, \ldots, m
\end{array}\right\} \Longrightarrow \begin{cases}\lambda_{j}^{\nu}=0, & j \in \gamma_{\infty}^{\nu} \\
\mu_{i}^{\nu}=0, & i=1, \ldots, m\end{cases}
$$

where $\gamma_{\infty}^{\nu}:=\left\{j \mid h_{j}^{\nu}\left(x_{\infty}^{\nu}\right)=0\right\} \subseteq\left\{1,2, \ldots, l_{\nu}\right\}$. Then $x_{\infty}$ is a solution of GNEP P.

Proof. From Corollary 5.1, $x_{k}$ is a solution of the following QVI: Find $x \in S_{0}(x)$ such that

$$
\left\langle F(x)-\rho_{k} E(x), y-x\right\rangle \geq 0, \quad \forall y \in S_{0}(x) .
$$

Let $\left\{x_{k}\right\}_{k \in \kappa}$ be a convergent subsequence whose limit is $x_{\infty}$. By Assumption 5.2, the linear independence constraint qualification holds for problem $P_{\rho_{k}}$. Thus, it follows from the KarushKuhn-Tucker (KKT) condition for problem (19) that for any $k$ there exist Lagrange multipliers

\footnotetext{
${ }^{1}$ In [12], the concept of diagonal strict concavity is introduced, since each player is supposed to solve a maximization problem.
} 
$\left(\mu_{k, i}^{\nu}\right)_{i=1}^{m}$ such that

$$
F^{\nu}\left(x_{k}\right)-\rho_{k} \sum_{j=1}^{l_{\nu}} \frac{1}{h_{j}^{\nu}\left(x_{k}^{\nu}\right)} \nabla h_{j}^{\nu}\left(x_{k}^{\nu}\right)+\sum_{i=1}^{m} \mu_{k, i}^{\nu} a_{i}^{\nu}=0, \quad \nu=1, \ldots, N .
$$

Put $\lambda_{k, j}^{\nu}:=-\rho_{k} / h_{j}^{\nu}\left(x_{k}^{\nu}\right) \geq 0, j=1, \ldots, l_{\nu}$, and define the vectors

$$
\phi_{k}^{\nu}:=\left(\begin{array}{c}
\lambda_{k}^{\nu} \\
\mu_{k}^{\nu}
\end{array}\right)
$$

where $\lambda_{k}^{\nu}:=\left(\lambda_{k, j}^{\nu}\right)_{j=1}^{l_{\nu}}$ and $\mu_{k}^{\nu}:=\left(\mu_{k, i}^{\nu}\right)_{i=1}^{m}$. Let us show that the sequence $\left\{\phi_{k}^{\nu}\right\}_{k \in \kappa}$ is bounded for each $\nu$. In fact, if $\left\{\phi_{k}^{\nu}\right\}_{k \in \kappa}$ is unbounded, then there exists a further subsequence $\left\{\phi_{k}^{\nu}\right\}_{k \in \kappa^{\prime}}$ such that

$$
\lim _{\kappa^{\prime} \ni k \rightarrow \infty}\left\|\phi_{k}^{\nu}\right\|=\infty .
$$

By dividing both sides of (20) by $\left\|\phi_{k}^{\nu}\right\|$, we have

$$
\frac{1}{\left\|\phi_{k}^{\nu}\right\|} F^{\nu}\left(x_{k}\right)+\sum_{j=1}^{l_{\nu}} \frac{\lambda_{k, j}^{\nu}}{\left\|\phi_{k}^{\nu}\right\|} \nabla h_{j}^{\nu}\left(x_{k}^{\nu}\right)+\sum_{i=1}^{m} \frac{\mu_{k, i}^{\nu}}{\left\|\phi_{k}^{\nu}\right\|} a_{i}^{\nu}=0, \quad \nu=1, \ldots, N .
$$

Since $\left\{\lambda_{k, j}^{\nu} /\left\|\phi_{k}^{\nu}\right\|\right\}_{k \in \kappa^{\prime}}$ and $\left\{\mu_{k, i}^{\nu} /\left\|\phi_{k}^{\nu}\right\|\right\}_{k \in \kappa^{\prime}}$ are bounded, these sequences have accumulation points $\bar{\lambda}_{j}^{\nu}$ and $\bar{\mu}_{i}^{\nu}$, respectively. Therefore, we have

$$
\sum_{j=1}^{l_{\nu}} \bar{\lambda}_{j}^{\nu} \nabla h_{j}^{\nu}\left(x_{\infty}^{\nu}\right)+\sum_{i=1}^{m} \bar{\mu}_{i}^{\nu} a_{i}^{\nu}=0, \quad \nu=1, \ldots, N .
$$

Now notice that $\bar{\lambda}_{j}^{\nu} \geq 0$ for all $j$. In particular, since

$$
\limsup _{k \rightarrow \infty} h_{j}^{\nu}\left(x_{k}^{\nu}\right)<0, \quad \forall j \notin \gamma_{\infty}^{\nu},
$$

we have $\lambda_{k, j}^{\nu}=0$, for all $k \in \kappa^{\prime}$ sufficient large, implying $\bar{\lambda}_{j}^{\nu}=0$ for all $j \notin \gamma_{\infty}^{\nu}$. Thus, it follows from (21) that

$$
\sum_{j \in \gamma_{\infty}^{\nu}} \bar{\lambda}_{j}^{\nu} \nabla h_{j}^{\nu}\left(x_{\infty}^{\nu}\right)+\sum_{i=1}^{m} \bar{\mu}_{i}^{\nu} a_{i}^{\nu}=0, \quad \nu=1, \ldots, N .
$$

However, this along with the fact that

$$
\bar{\lambda}_{j}^{\nu} \geq 0, \quad \forall j \in \gamma_{\infty}^{\nu}, \quad \text { and } \quad\left\|\left(\begin{array}{c}
\bar{\lambda}^{\nu} \\
\bar{\mu}^{\nu}
\end{array}\right)\right\|=1
$$

contradicts the assumed MFCQ. This implies that $\left\{\phi_{k}^{\nu}\right\}$ is bounded, and that $\left\{\lambda_{k}^{\nu}\right\}$ and $\left\{\mu_{k}^{\nu}\right\}$ have accumulation points $\lambda_{\infty}^{\nu}$ and $\mu_{\infty}^{\nu}$, respectively. Therefore, $x_{\infty}$ satisfies

$$
\left.\begin{array}{l}
F^{\nu}\left(x_{\infty}\right)+\sum_{j=1}^{l_{\nu}} \lambda_{\infty, j}^{\nu} \nabla h_{j}^{\nu}\left(x_{\infty}^{\nu}\right)+\sum_{i=1}^{m} \mu_{i}^{\nu} a_{i}^{\nu}=0 \\
h_{j}^{\nu}\left(x_{\infty}^{\nu}\right) \leq 0, \quad \lambda_{\infty, j}^{\nu} \geq 0, \quad \lambda_{\infty, j}^{\nu} h_{j}^{\nu}\left(x_{\infty}^{\nu}\right)=0, \quad j=1, \ldots, l_{\nu} \\
\left\langle a_{i}^{\nu}, x_{\infty}^{\nu}\right\rangle+\sum_{\nu^{\prime} \neq \nu}\left\langle a_{i}^{\nu^{\prime}}, x_{\infty}^{\nu^{\prime}}\right\rangle-b_{i}=0, \quad i=1, \ldots, m
\end{array}\right\}, \quad \nu=1, \ldots, N .
$$


This is nothing but the KKT condition for problem (3) with $S(x)$ defined by the constraints in problems (5). Consequently, $x_{\infty}$ is a solution of GNEP $P$.

\section{Extension to GNEP with Shared Inequality Constraints}

The GNEP considered in the previous section assumes that each player's shared constraints are defined by equalities only. In practice, however, the shared constraints often contain inequalities. In this section, we discuss the case of shared linear inequality constraints and present an approach that relies on the transformation to the equality constraints by means of slack variables.

Suppose that, for each $\nu$, player $\nu$ 's problem is given as

$$
\begin{array}{cl}
\underset{x^{\nu}}{\operatorname{minimize}} & \theta^{\nu}\left(x^{\nu}, x^{-\nu}\right) \\
\text { subject to } & \left\langle a_{i}^{\nu}, x^{\nu}\right\rangle \leq b_{i}-\sum_{\nu^{\prime} \neq \nu}\left\langle a_{i}^{\nu^{\prime}}, x^{\nu^{\prime}}\right\rangle, \quad i=1, \ldots, m, \\
& h_{j}^{\nu}\left(x^{\nu}\right) \leq 0, \quad j=1, \ldots, l_{\nu} .
\end{array}
$$

Denote this GNEP as $\hat{P}$. Introducing slack variables $s^{\nu}:=\left(s_{1}^{\nu}, \ldots, s_{m}^{\nu}\right)$ as supplementary variables for each player $\nu$, problem (22) is rewritten as

$$
\begin{array}{cl}
\underset{x^{\nu}, s^{\nu}}{\operatorname{minimize}} & \theta^{\nu}\left(x^{\nu}, x^{-\nu}\right) \\
\text { subject to } & \left\langle a_{i}^{\nu}, x^{\nu}\right\rangle+s_{i}^{\nu}=b_{i}-\sum_{\nu^{\prime} \neq \nu}\left(\left\langle a_{i}^{\nu^{\prime}}, x^{\nu^{\prime}}\right\rangle+s_{i}^{\nu^{\prime}}\right), \quad i=1, \ldots, m, \\
& h_{j}^{\nu}\left(x^{\nu}\right) \leq 0, \quad j=1, \ldots, l_{\nu} \\
& s_{i}^{\nu} \geq 0, \quad i=1, \ldots, m
\end{array}
$$

Denote this GNEP as $\check{P}$. The vector consisting of all slack variables is denoted by $s:=$ $\left(s^{\nu}\right)_{\nu=1}^{N} \in \mathbb{R}^{N m}$. The next result shows that, under some conditions, a solution of GNEP $\check{P}$ is also a solution of GNEP $\hat{P}$.

Theorem 7.1. Let $(x, s)$ be a solution of GNEP $\check{P}$. If the relation

$$
s_{i}^{\nu}=0 \text { for some } \nu \quad \Longrightarrow \quad s_{i}^{\nu}=0 \text { for all } \nu
$$

holds for all $i=1, \ldots, m$, then $x$ is a solution of GNEP $\hat{P}$.

Proof. Define the Lagrangian for problem (23) by

$$
\begin{aligned}
\mathcal{L}^{\nu}\left(x^{\nu}, s^{\nu}, \lambda^{\nu}, \mu^{\nu}, \eta^{\nu}\right):= & \theta^{\nu}\left(x^{\nu}, x^{-\nu}\right)+\sum_{i=1}^{m} \mu_{i}^{\nu}\left(\left\langle a_{i}^{\nu}, x^{\nu}\right\rangle+s_{i}^{\nu}+\sum_{\nu^{\prime} \neq \nu}\left\langle a_{i}^{\nu^{\prime}}, x^{\nu^{\prime}}\right\rangle+\sum_{\nu^{\prime} \neq \nu} s_{i}^{\nu^{\prime}}-b_{i}\right) \\
& +\sum_{j=1}^{l_{\nu}} \lambda_{j}^{\nu} h_{j}^{\nu}\left(x^{\nu}\right)-\sum_{i=1}^{m} \eta_{i}^{\nu} s_{i}^{\nu} .
\end{aligned}
$$


A solution $(x, s)$ of GNEP $\check{P}$ satisfies the following $\mathrm{KKT}$ conditions for all $\nu$ :

$$
\begin{aligned}
& \nabla_{x^{\nu}} \mathcal{L}^{\nu}\left(x^{\nu}, s^{\nu}, \lambda^{\nu}, \mu^{\nu}, \eta^{\nu}\right)=\nabla_{x^{\nu}} \theta^{\nu}\left(x^{\nu}, x^{-\nu}\right)+\sum_{i=1}^{m} \mu_{i}^{\nu} a_{i}^{\nu}+\sum_{j=1}^{l_{\nu}} \lambda_{j}^{\nu} \nabla h_{j}^{\nu}\left(x^{\nu}\right)=0, \\
& \nabla_{s^{\nu}} \mathcal{L}^{\nu}\left(x^{\nu}, s^{\nu}, \lambda^{\nu}, \mu^{\nu}, \eta^{\nu}\right)=\mu^{\nu}-\eta^{\nu}=0, \\
& \left\langle a_{i}^{\nu}, x^{\nu}\right\rangle+s_{i}^{\nu}+\sum_{\nu^{\prime} \neq \nu}\left\langle a_{i}^{\nu^{\prime}}, x^{\nu^{\prime}}\right\rangle+\sum_{\nu^{\prime} \neq \nu} s_{i}^{\nu^{\prime}}-b_{i}=0, \quad i=1, \ldots, m, \\
& \lambda_{j}^{\nu} \geq 0, \quad \lambda_{j}^{\nu} h_{j}^{\nu}\left(x^{\nu}\right)=0, \quad h_{j}^{\nu}\left(x^{\nu}\right) \leq 0, \quad j=1, \ldots, l_{\nu}, \\
& \eta_{i}^{\nu} \geq 0, \quad \eta_{i}^{\nu} s_{i}^{\nu}=0, \quad s_{i}^{\nu} \geq 0, \quad i=1, \ldots, m .
\end{aligned}
$$

By the relation (24), we have for each $i$ either (i) $s_{i}^{\nu}=0$ for all $\nu$, or (ii) $s_{i}^{\nu}>0$ for all $\nu$. Let us consider these two cases separately.

(i) Suppose $s_{i}^{\nu}=0$ for all $\nu$. By $(25 \mathrm{e})$, we have

$$
\eta_{i}^{\nu} \geq 0
$$

Then it follows from $(25 \mathrm{~b})$ and $(25 \mathrm{c})$ that

$$
\mu_{i}^{\nu} \geq 0
$$

and

$$
\left\langle a_{i}^{\nu}, x^{\nu}\right\rangle+\sum_{\nu^{\prime} \neq \nu}\left\langle a_{i}^{\nu^{\prime}}, x^{\nu^{\prime}}\right\rangle-b_{i}=0
$$

for all $\nu$.

(ii) Suppose $s_{i}^{\nu}>0$ for all $\nu$. Then, by (25e), we have

$$
\eta_{i}^{\nu}=0 \text {. }
$$

Therefore, from (25b) and (25c), we obtain

$$
\mu_{i}^{\nu}=0
$$

and

$$
\left\langle a_{i}^{\nu}, x^{\nu}\right\rangle+\sum_{\nu^{\prime} \neq \nu}\left\langle a_{i}^{\nu^{\prime}}, x^{\nu^{\prime}}\right\rangle-b_{i}<0
$$

for all $\nu$.

Hence, the following complementarity conditions hold for all $i$ :

$$
\mu_{i}^{\nu} \geq 0, \quad \mu_{i}^{\nu}\left(\sum_{\nu=1}^{N}\left\langle a_{i}^{\nu}, x^{\nu}\right\rangle-b_{i}\right)=0, \quad \sum_{\nu=1}^{N}\left\langle a_{i}^{\nu}, x^{\nu}\right\rangle-b_{i} \leq 0 .
$$

Combining (25a), (25d) and (26), we have for all $\nu$

$$
\begin{aligned}
& \nabla_{x^{\nu}} \theta^{\nu}\left(x^{\nu}, x^{-\nu}\right)+\sum_{i=1}^{m} \mu_{i}^{\nu} a_{i}^{\nu}+\sum_{j=1}^{l_{\nu}} \lambda_{j}^{\nu} \nabla h_{j}^{\nu}\left(x^{\nu}\right)=0, \\
& \mu_{i}^{\nu} \geq 0, \quad \mu_{i}^{\nu}\left(\sum_{\nu=1}^{N}\left\langle a_{i}^{\nu}, x^{\nu}\right\rangle-b_{i}\right)=0, \quad \sum_{\nu=1}^{N}\left\langle a_{i}^{\nu}, x^{\nu}\right\rangle-b_{i} \leq 0, \quad i=1, \ldots, m, \\
& \lambda_{j}^{\nu} \geq 0, \quad \lambda_{j}^{\nu} h_{j}^{\nu}\left(x^{\nu}\right)=0, \quad h_{j}^{\nu}\left(x^{\nu}\right) \leq 0, \quad j=1, \ldots, l_{\nu} .
\end{aligned}
$$


This implies that for each $\nu, x^{\nu}$ satisfies the KKT condition for problem (22) with given $x^{-\nu}$. Thus, $x=\left(x^{\nu}\right)_{\nu=1}^{N}$ is a solution of GNEP $\hat{P}$.

By adding the barrier term associated with the individual constraints to the objective function, player $\nu$ 's problem (23) is approximated by the following problem:

$$
\begin{array}{ll}
\underset{x^{\nu}, s^{\nu}}{\operatorname{minimize}} & \theta^{\nu}\left(x^{\nu}, x^{-\nu}\right)-\rho\left(\sum_{j=1}^{l_{\nu}} \log \left(-h_{j}^{\nu}\left(x^{\nu}\right)\right)+\sum_{i=1}^{m} \log s_{i}^{\nu}\right) \\
\text { subject to } & \left\langle a_{i}^{\nu}, x^{\nu}\right\rangle+s_{i}^{\nu}=b_{i}-\sum_{\nu^{\prime} \neq \nu}\left(\left\langle a_{i}^{\nu^{\prime}}, x^{\nu^{\prime}}\right\rangle+s_{i}^{\nu^{\prime}}\right), \quad i=1, \ldots, m .
\end{array}
$$

Denote this GNEP as $\check{P}_{\rho}$. Let the function $\check{F}: \mathbb{R}^{n+N m} \rightarrow \mathbb{R}^{n+N m}$ be defined by

$$
\check{F}(x, s):=\left(\begin{array}{c}
F(x) \\
0
\end{array}\right) \in \mathbb{R}^{n+N m}
$$

where $F(x)$ is given by (2). Define the function $G: \mathbb{R}^{N m} \rightarrow \mathbb{R}^{N m}$ by

$$
G(s):=\left(\left(1 / s_{i}^{\nu}\right)_{i=1}^{m}\right)_{\nu=1}^{N} .
$$

Moreover, let the function $\check{E}: \mathbb{R}^{n+N m} \rightarrow \mathbb{R}^{n+N m}$ be defined by

$$
\check{E}(x, s):=\left(\begin{array}{c}
E(x) \\
G(s)
\end{array}\right) \in \mathbb{R}^{n+N m}
$$

where $E(x)$ is given by (8).

Since problem $(27)$ is a convex programming problem, GNEP $\check{P}_{\rho}$ can be reformulated as the following QVI: Find $(x, s) \in \check{S}_{0}(x, s) \cap \check{\Sigma}_{0}$ such that

$$
\langle\check{F}(x, s)-\rho \check{E}(x, s),(y, t)-(x, s)\rangle \geq 0, \quad \forall(y, t) \in \check{S}_{0}(x, s),
$$

where $\check{\Sigma}_{0} \subseteq \mathbb{R}^{n+N m}$ and $\check{S}_{0}: \mathbb{R}^{n+N m} \rightrightarrows \mathbb{R}^{n+N m}$ are defined by

$$
\begin{aligned}
\check{\Sigma}_{0} & :=\prod_{\nu=1}^{N}\left\{\left(x^{\nu}, s^{\nu}\right) \mid h_{j}^{\nu}\left(x^{\nu}\right)<0, j=1, \ldots, l_{\nu}, s_{i}^{\nu}>0, i=1, \ldots, m\right\}, \\
\check{S}_{0}(x, s) & :=\prod_{\nu=1}^{N}\left\{\left(y^{\nu}, t^{\nu}\right) \mid\left\langle a_{i}^{\nu}, y^{\nu}\right\rangle+t_{i}^{\nu}=b_{i}-\sum_{\nu^{\prime} \neq \nu}\left\langle a_{i}^{\nu^{\prime}}, x^{\nu^{\prime}}\right\rangle-\sum_{\nu^{\prime} \neq \nu} s_{i}^{\nu^{\prime}}, i=1, \ldots, m\right\},
\end{aligned}
$$

respectively. Then, the regularized gap function for this problem is given by

$$
\begin{aligned}
\check{f}_{\rho}(x, s):=-\inf \{ & \langle\check{F}(x, s)-\rho \check{E}(x, s),(y, t)-(x, s)\rangle \\
& \left.+\frac{1}{2}\langle(y, t)-(x, s), \check{H}((y, t)-(x, s))\rangle \mid(y, t) \in \check{S}_{0}(x, s)\right\},
\end{aligned}
$$


where $\check{H}$ is a symmetric positive definite matrix. Note that the function $\check{f}_{\rho}$ is defined only on the open set $\check{\Sigma}_{0}$. By letting $\check{f}_{\rho}(x, s):=+\infty \forall(x, s) \notin \check{\Sigma}_{0}$, GNEP $\check{P}_{\rho}$ is reformulated as the minimization problem

$$
\begin{array}{lll}
\check{Q}_{\rho}: \quad & \text { minimize } & \check{f}_{\rho}(x, s) \\
& \text { subject to } & (x, s) \in \check{X}_{0},
\end{array}
$$

where the set $\check{X}_{0} \subseteq \mathbb{R}^{n+N m}$ is defined by

$$
\check{X}_{0}:=\left\{(x, s) \mid b_{i}-\sum_{\nu=1}^{N}\left\langle a_{i}^{\nu}, x^{\nu}\right\rangle-\sum_{\nu=1}^{N} s_{i}^{\nu}=0, i=1, \ldots, m\right\} .
$$

This fact implies that for each $\rho>0$, the set of optimum solutions of problem $\check{Q}_{\rho}$ equals the set of solutions of GNEP $\check{P}_{\rho}$. We will then show that any stationary point for problem $\check{Q}_{\rho}$ is a solution of GNEP $\check{P}_{\rho}$ under some conditions.

Lemma 7.1. Suppose $x \in \Sigma_{0}, s>0$ and $\rho>0$. Let $\nabla F(x)-\rho \nabla E(x)$ be positive definite. Then, $\nabla \check{F}(x, s)-\rho \nabla \check{E}(x, s)$ is also positive definite.

Proof. By direct calculation, we have

$$
\nabla G(s)=\operatorname{diag}\left(\left(-1 /\left(s_{i}^{\nu}\right)^{2}\right)_{i=1}^{m}\right)_{\nu=1}^{N},
$$

which is a negative definite matrix. Thus, by the given assumption, for all $\xi \in \mathbb{R}^{n}$ and $\sigma \in \mathbb{R}^{N m}$ such that $(\xi, \sigma) \neq(0,0)$, it follows that

$$
\begin{aligned}
(\xi, \sigma)^{\top}(\nabla \check{F}(x, s)-\rho \nabla \check{E}(x, s))(\xi, \sigma) & =(\xi, \sigma)^{\top}\left(\left(\begin{array}{cc}
\nabla F(x) & 0 \\
0 & 0
\end{array}\right)-\rho\left(\begin{array}{cc}
\nabla E(x) & 0 \\
0 & \nabla G(s)
\end{array}\right)\right)(\xi, \sigma) \\
& =\xi^{\top}(\nabla F(x)-\rho \nabla E(x)) \xi+\sigma^{\top}(-\rho \nabla G(s)) \sigma \\
& >0
\end{aligned}
$$

for $\rho>0$. This completes the proof.

Theorem 7.2. Suppose Assumption 5.2 holds. Let $(x, s) \in \check{X}_{0}$ be a stationary point of problem $\check{Q}_{\rho}$, and $\nabla F(x)-\rho \nabla E(x)$ be positive definite for $\rho>0$. Then the point $(x, s)$ is a solution of $Q V I(28)$, i.e., $(x, s)$ is a solution of GNEP $\check{P}_{\rho}$.

Proof. By Assumption 5.2, the vectors $a_{i}^{\nu}, i=1, \ldots, m$ are linearly independent for each $\nu=1, \ldots, N$. Therefore, the vectors $\left(\left(0, \ldots, 0, a_{i}^{\nu}, 0, \ldots, 0\right)^{\top}, e_{i}^{\nu}\right) \in \mathbb{R}^{n+N m}, i=1, \ldots, m, \nu=$ $1, \ldots, N$ are linearly independent, where $e_{i}^{\nu} \in \mathbb{R}^{N m}$ denotes the unit vector whose element corresponding to $s_{i}^{\nu}$ is one and the others are zero. Thus, the following set is a singleton:

$$
\begin{aligned}
\check{M}(x, s):=\left\{\mu \in \mathbb{R}^{N m} \mid \check{F}(x, s)-\rho \check{E}(x, s)+\check{H}((y(x, s), t(x, s))-(x, s))\right. \\
\left.+\sum_{i=1}^{m} \sum_{\nu=1}^{N} \mu_{i}^{\nu}\left(\left(0, \ldots, 0, a_{i}^{\nu}, 0, \ldots, 0\right)^{\top}, e_{i}^{\nu}\right)=0\right\} .
\end{aligned}
$$


That is, $\check{M}(x, s)$ has only one element $\mu(x, s)$. Hence, by Theorem 5.2 , the function $\check{f}_{\rho}$ is differentiable at any point $(x, s) \in \check{\Sigma}_{0}$.

Since $\nabla F(x)-\rho \nabla E(x)$ is positive definite, we have that $\nabla \check{F}(x, s)-\rho \nabla \check{E}(x, s)$ is positive definite by Lemma 7.1. Therefore, Theorem 5.4 ensures that any stationary point $(x, s)$ of problem $\check{Q}_{\rho}$ solves QVI $(28)$, which means $(x, s)$ is a solution of GNEP $\check{P}_{\rho}$.

To solve the minimization problem $\check{Q}_{\rho}$, we may apply an SQP-type iterative method using the gradient values of the function $\check{f}_{\rho}$. Moreover, in the hope that condition (24) is satisfied at the computed solution of problem $\check{Q}_{\rho}$, we add the following correction step at every iteration of the SQP method: For every $i$,

$$
\text { if } s_{i}^{\nu}<\varepsilon \text { for some } \nu \text {, then put } s_{i}^{\nu}:=\frac{1}{N} \sum_{\nu=1}^{N} s_{i}^{\nu} \text { for all } \nu \text {. }
$$

Although this modification does not change the value of the sum $\sum_{\nu=1}^{N} s_{i}^{\nu}$ for each $i$, and hence all the equality constraints

$$
b_{i}-\sum_{\nu=1}^{N}\left\langle a_{i}^{\nu}, x^{\nu}\right\rangle-\sum_{\nu=1}^{N} s_{i}^{\nu}=0, \quad i=1, \ldots, m
$$

remain to be satisfied, it may affect the theoretical convergence property of the SQP method. However, as far as our numerical experiments reported in the next section are concerned, the method always generated a sequence converging to a stationary point of problem $\check{Q}_{\rho}$.

An algorithm for finding a solution of GNEP $\hat{P}$ may be stated as follows:

Algorithm 7.1. Choose a positive sequence $\left\{\rho_{k}\right\} \subseteq \mathbb{R}$ tending to zero. For each $k$, solve the minimization problem $\check{Q}_{\rho_{k}}$, and proceed to the next step by increasing $k$ by one.

We may expect that this algorithm yields a solution to GNEP $\hat{P}$, because the correction step (29) used in the SQP method for solving each problem $\check{Q}_{\rho_{k}}$ would help condition (24) in Theorem 7.1 to hold in the limit. However, unlike Algorithm 6.1, it seems to be difficult to establish theoretical convergence to a solution of problem $\check{P}$ satisfying condition (24) even if this condition holds at a solution of every $\check{Q}_{\rho_{k}}$. Nevertheless, in our numerical experiments to be reported in the next section, the correction step (29) used in the SQP method worked quite successfully and solutions of GNEP $\check{P}$ satisfying condition (24) were obtained in almost all cases.

\section{Numerical Results}

In this section, we report our numerical experience with Algorithm 7.1 for some examples. The matrix $\check{H}$ in the definition of the function $\check{f}_{\rho}$ is chosen to be the identity matrix. Algorithn 7.1 is terminated when the barrier parameter $\rho_{k}$ becomes less than $10^{-15}$. To solve minimization problems $\check{Q}_{\rho_{k}}$, we use an SQP method incorporating the correction step (29) with $\varepsilon=10^{-7}$. All programs are coded in MatLAB. 
Example 1. We consider Harker's example [17]. In this game, there are two players who solve the following problems:

$$
\begin{array}{cll} 
& \underset{x^{1}}{\operatorname{minimize}} & \left(x^{1}\right)^{2}+(8 / 3) x^{1} x^{2}-34 x^{1} \\
P_{1}\left(x^{2}\right): \quad \text { subject to } & 0 \leq x^{1} \leq 10, \\
& x^{1}+x^{2} \leq 15 . \\
& \\
& \\
P_{2}\left(x^{1}\right): \quad \text { minimize } & \left(x^{2}\right)^{2}+(5 / 4) x^{1} x^{2}-24.25 x^{2} \\
& \text { subject to } & 0 \leq x^{2} \leq 10, \\
& & x^{1}+x^{2} \leq 15 .
\end{array}
$$

The solution set of this GNEP is known to be

$$
\left\{\left(\begin{array}{l}
5 \\
9
\end{array}\right)\right\} \cup\left\{\left(\begin{array}{c}
t \\
15-t
\end{array}\right) \mid 9 \leq t \leq 10\right\} .
$$

We have tried various starting points and barrier parameters in implementing Algorithm 7.1, and then observed that a generated sequence always converged to the particular GNE $x=$ $(5,9)^{\top}$.

Example 2. We consider the three-person river basin pollution game [6]. In this game, the problem of each player $\nu \in\{1,2,3\}$ is given by

$$
\begin{aligned}
\operatorname{minimize} & \left(c_{1 \nu}+c_{2 \nu} x^{\nu}\right) x^{\nu}-\left(d_{1}-d_{2}\left(x^{1}+x^{2}+x^{3}\right)\right) x^{\nu} \\
P^{\nu}\left(x^{-\nu}\right): \quad \text { subject to } & \sum_{\nu=1}^{3} u_{\nu l} e_{\nu} x^{\nu} \leq K_{l}, \quad l=1,2 \\
& x^{\nu} \geq 0,
\end{aligned}
$$

where $d_{1}=3.0, d_{2}=0.01, K_{l}=100, l=1,2$, and the other constants are shown in Table 1 .

Table 1: Problem data for the river basin pollution game.

\begin{tabular}{cccccc}
\hline Player $\nu$ & $c_{1 \nu}$ & $c_{2 \nu}$ & $e_{\nu}$ & $u_{\nu 1}$ & $u_{\nu 2}$ \\
\hline 1 & 0.10 & 0.01 & 0.50 & 6.5 & 4.583 \\
2 & 0.12 & 0.05 & 0.25 & 5.0 & 6.250 \\
3 & 0.15 & 0.01 & 0.75 & 5.5 & 3.750 \\
\hline
\end{tabular}

We implemented Algorithm 7.1 by using 10000 starting points randomly generated in the feasible set, and found many different GNEs as shown in Figure 1.

Example 3. We consider the internet switching model [22]. In this game, the problem of each 


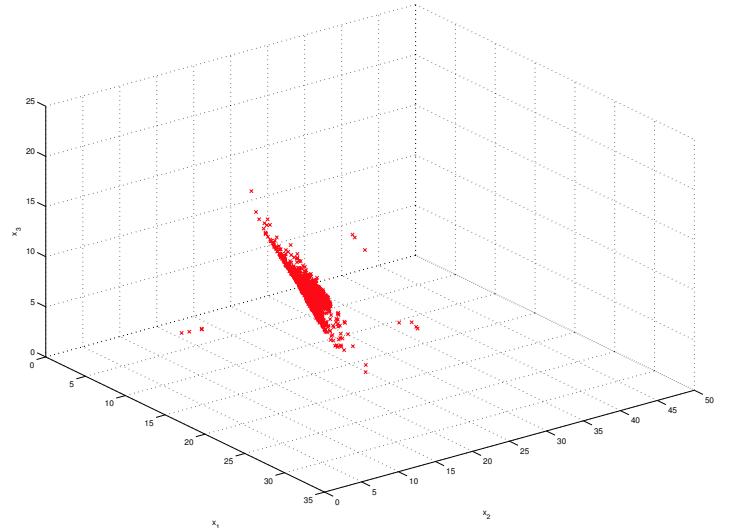

Figure 1: GNEs of the river basin pollution game found by Algorithm 7.1.

player $\nu \in\{1, \ldots, N\}$ is given by

$$
\begin{aligned}
\operatorname{minimize} & \theta^{\nu}\left(x^{\nu}, x^{-\nu}\right)=\frac{x^{\nu}}{B}-\frac{x^{\nu}}{\sum_{\nu=1}^{N} x^{\nu}} \\
P^{\nu}\left(x^{-\nu}\right): \quad \text { subject to } & \sum_{\nu=1}^{N} x^{\nu} \leq B, \\
& x^{\nu} \geq 0.01 .
\end{aligned}
$$

We set $N=10$ and $B=1$. Using 100 starting points randomly generated in the set $\Sigma_{0}:=$ $\left\{x \mid \sum_{\nu=1}^{N} x^{\nu}<B, x^{\nu}>0.01, \nu=1, \ldots, N\right\}$, we implemented Algorithm 7.1 and always obtained the point $x=(0.09,0.09, \ldots, 0.09)^{\top}$, which is the unique solution of the GNEP [22].

\section{Conclusion}

We have proposed a gap function approach to the GNEP in which the shared constraints are given by linear equalities, while the individual constraints are given by convex inequalities. We apply a barrier technique to individual inequality constraints and transform each player's problem into a problem involving the shared equality constraints only. Further, we have shown that the proposed approach can be extended to GNEPs with shared linear inequality constraints by means of slack variables. We have implemented the proposed sequential minimization method on some examples and confirmed that the method can find a solution of those problems.

As mentioned in Introduction, there have been proposed several methods for solving GNEPs, with different problem settings and different purposes, e.g., (i) computing an arbitrary GNE, (ii) computing all or as many as GNEs, or (iii) computing a particular GNE such as a normalized equilibrium. The method proposed in this paper can be categorized as a method of type (ii), although the applicable class of problems is limited to those with linear shared constraints. In this category, the only method known to the authors so far is the method proposed in [15] that solves a parametrized family of VIs, each of which yields a GNE. By comparing the numerical 
results reported in [15] and this paper on the same examples, we may observe that the method of [15] is able to obtain more widely distributed GNEs. However the parametrization mechanism used in [15] is somewhat involved and the gap function approach proposed in this paper has the advantage that it is simpler to implement in practice.

Acknowledgement. This work was supported in part by the Scientific Research Grant-in-Aid from the Japan Society for the Promotion of Science. The authors are grateful to Professor Franco Giannessi and an anonymous referee for their helpful comments.

\section{References}

[1] Facchinei, F., Kanzow, C.: Generalized Nash equilibrium problems, 4OR: A Quarterly Journal of Operations Research 5, 173-210 (2007).

[2] HobBs, B.F., PANG, J.S.: Nash-Cournot equilibrium in electric power markets with piecewise linear demand functions and joint constraints, Operations Research 55, 113-127 (2007).

[3] Pang, J.S., Fukushima, M.: Quasi-variational inequalities, generalized Nash equilibria, and multi-leader-follower games, Computational Management Science 2, 21-56 (2005); Erratum, ibid. 6, 373-375 (2009).

[4] Haurie, A., Krawczyk, J.B.: Optimal charges on river effluent from lumped and distributed sources, Environmental Modeling and Assessment 2, 93-106 (1997).

[5] KrawczyK, J.B.: Coupled constraint Nash equilibria in environmental games, Resource and Energy Economics 27, 157-181 (2005).

[6] Krawczyk, J.B., Uryasev, S.: Relaxation algorithms to find Nash equilibria with economic applications, Environmental Modeling and Assessment 5, 63-73 (2000).

[7] Facchinei, F., Pang, J.S.: Finite-Dimensional Variational Inequalities and Complementarity Problems, Springer, New York (2003).

[8] Harker, P.T.: A variational inequality approach for the determination of oligopolistic market equilibrium, Mathematical Programming 30, 105-111 (1984).

[9] Harker, P.T., PANG, J.S.: Finite-dimensional variational inequality and nonlinear complementarity problems: A survey of theory, algorithms and applications, Mathematical Programming 48, 161-220 (1990).

[10] Facchinei, F., PAng, J.S.: Exact penalty functions for generalized Nash problems, in Large-Scale Nonlinear Optimization, Edited by G. Di Pillo and M. Roma, pp. 115-126, Springer, Heidelberg (2006). 
[11] Fukushima, M.: Restricted generalized Nash equilibria and controlled penalty algorithm, Computational Management Science (to appear).

[12] Rosen, J.B.: Existence and uniqueness of equilibrium points for concave $N$-person games, Econometrica 33, 520-534 (1965).

[13] Facchinei, F., Fischer, A., Piccialli, P.: Generalized Nash equilibrium problems and Newton methods, Mathematical Programming 117, 163-194 (2009).

[14] von Heusinger, A., Kanzow, C., Fukushima, M.: Newton's method for computing a normalized equilibrium in the generalized Nash game through fixed point formulation, Technical Report 2009-006, Department of Applied Mathematics and Physics, Kyoto University (2009).

[15] Nabetani, K., Tseng, P., Fukushima, M.: Parametrized variational inequality approaches to generalized Nash equilibrium problems with shared constraints, Computational Optimization and Applications (to appear).

[16] von Heusinger, A., Kanzow, C.: Optimization reformulations of the generalized Nash equilibrium problem using Nikaido-Isoda-type functions, Computational Optimization and Applications 43, 353-377 (2009).

[17] Harker, P.T.: Generalized Nash games and quasi-variational inequalities, European Journal of Operational Research 54, 81-94 (1991).

[18] Bensoussan, A.: Points de Nash dans le cas de fontionnelles quadratiques et jeux differentiels linéaires a $n$ personnes, SIAM Journal on Control 12, 460-499 (1974).

[19] Fukushima, M.: A class of gap functions for quasi-variational inequality problems, Journal of Industrial and Management Optimization 3, 165-171 (2007).

[20] Fukushima, M.: Equivalent differentiable optimization problems and descent methods for asymmetric variational inequality problems, Mathematical Programming 53, 99-110 (1992).

[21] Auslender, A.: Optimisation: Méthodes Numériques, Masson, Paris (1976).

[22] Kesselman, A., Leonardi, S., Bonifaci, V.: Game-theoretic analysis of internet switching with selfish users, Lecture Notes in Computer Science 3828, 236-245 (2005). 